\title{
Offering HeLP to work out the optimum treatment for haemorrhoidal disease
}

\author{
Steven R. Brown ${ }^{1}$. Matthew J. Lee ${ }^{1}$
}

Received: 5 September 2018 / Accepted: 7 September 2018 / Published online: 14 September 2018

(c) Springer Nature Switzerland AG 2018

Haemorrhoidal disease is a gold mine for research and innovation. It is a very common condition, with a large population of potential study recruits. And, as yet, there is no perfect intervention-there is a gap in the market. The perfect surgical intervention should be appropriate for all grades of haemorrhoid, be easy to learn and perform, have durable clinical effectiveness, be tolerable to the patient, cause no harm, and be cost-effective. No intervention fully meets these criteria. Cheap and easy office procedures such as rubber band ligation may fail due to poor efficacy-recurrence or residual symptoms can occur in up to $50 \%$ of patients after a single procedure [1]. In contrast, more efficacious procedures such as conventional haemorrhoidectomy may be painful and often result in prolonged recovery [2]. Hence, a plethora of innovative techniques, in the last 20 years, is striving for this 'perfection'.

We have great admiration for the authors of the study published in this edition [3], not necessarily for the content of the report (which will be discussed later), but because we hope they are a part of the tiny minority of surgeons who fall into the bracket of innovators or 'true revolutionaries'. These are one of the six types of personality when it comes to innovation or revolution [4]. True revolutionaries think up an idea and push this idea into the surgical arena; if the procedure is a success, fame and fortune beckon, if a failure they sink back into obscurity. Like any true revolutionary, they are prepared to die for the cause! Two further groups may support an innovation. The 'true intellectuals' support only after careful scientific scrutiny and this tiny group are also to be admired. Not so admirable (and there are quite a few of them) are profiteers who will take up an innovation only for personal gain. If the procedure fails, they quickly move onto another opportunity. On the other side of the coin

Matthew J. Lee

m.j.lee@ sheffield.ac.uk

1 Department of General Surgery, Sheffield Teaching Hospitals, Sheffield S5 7AU, UK are the counter reactionaries who oppose and resist any innovation but will not necessarily preach their opposition. They are happy with the old order. 'It works for me, why change?' is their motto. Allied to this group are the counter revolutionaries. They will go all out to oppose any change, will be vocal about it and are prepared to die at the guillotine fighting change. There were several of these when it came to the PPH procedure. Finally there are the masses. They take up a new procedure if others do and a critical momentum is achieved. We admire the authors for what we hope is a 'revolutionary' stance (and Techniques in Coloproctology for giving many such 'revolutionaries' a voice); however, we must strive to be among the 'true intellectuals' and scientifically scrutinise this technique before taking it up ourselves.

The majority of the literature on haemorrhoidal disease, some 3000 papers in the last 25 years, can be criticised for similar reasons. Case series in single centres, poor definition of participants, no comparator, poorly defined outcome measures and an abbreviated follow-up period [5]. The authors highlight some of these drawbacks in their discussion. Nevertheless, they should be congratulated in coordinating a study involving 7 centres and recruiting nearly 300 patients. This in itself is no small task but to also achieve $100 \%$ follow-up at 6 months is truly extraordinary. We would love to know their secret.

Another positive of this trial is the attempt at standardising the technique and ensuring a learning curve has been defined and ascertained. Of course, it may be difficult to future proof a standard technique. PPH, DG HAL and even haemorrhoidectomy have undergone modifications to the 'standard technique' over the years with each published modification proclaiming superior results [6-8].

Enough of the positives. At least three of the highlighted limitations should be explored in more depth not only for scrutiny of this particularly intervention but to guide future innovation in haemorrhoidal disease.

Selection bias, particularly selection of less symptomatic patients, is always going to be an issue in any case series. 
Grade I piles and patients with acute haemorrhoidal symptoms (that may represent thrombosis) may not be appropriate indications for surgery. We would go further and suggest that, in general, surgeons operate on too many patients with haemorrhoids. Reassurance that symptoms are not sinister and a much stronger emphasis on conservative measures is certainly enough for grade I piles and probably for many with grade II piles or acute symptoms. There is a gathering movement (so to speak) for all grades of haemorrhoids to be treated more conservatively [9].

In our opinion by far the most pertinent limitation of this study and most other trials, including almost all of the $400+$ randomised controlled trials on haemorrhoidal disease, relates to the outcome measures. A recent work by van Tol has listed the 59 outcome measures that have been used in the literature, with a huge range of definitions, many having no definition at all [10]. This paper is no exception. It is essential to work out what is important for the patients to judge if the intervention has been successful or not. A core outcome set is being developed [11] and, provided it involves patients throughout, must be used for all future trials. Along these lines, a universally accepted and validated severity score and a simple but robust definition of failure or recurrence should also be used (we favour that suggested by Shanmugan et al.) [12]. Without these carefully defined, core patient-reported outcome measures, preferably used in a randomised controlled setting, a success rate of over $90 \%$ should be treated with healthy scepticism.

The final issue is often the anathema of any innovation, and that is cost. Whilst probably always important, it is becoming increasingly so. Recently, the UK government has sought to limit interventions for haemorrhoidal surgery, predicting huge savings to the NHS [13]. The authors imply a modest cost of 400 euros for HeLP, similar to the cost of a DGHAL, but with potential savings as anaesthesia is not usually required. The only way this can be proven to be costeffective is with a comparative trial. If, for instance, rubber band ligation was the comparator, the efficacy of HeLP would have to be significantly greater to offset the 'modest' cost and what is a substantially longer operative time.

Despite these specific criticisms, it is important not to stifle the ideal of innovation. As Einstein famously said. "If you always do what you've always done, you'll always get what you've always got". Long live the revolutionaries!

\section{Compliance with ethical standards}

Conflict of interest The authors declare that they have no conflict of interest.
Ethical approval For this type of manuscript, ethical approval is not required.

\section{References}

1. Brown SR, Tiernan JP, Watson AJM et al (2016) Haemorrhoidal artery ligation versus rubber band ligation for the management of symptomatic second-degree and third-degree haemorrhoids (HubBLe): a multicentre, open-label, randomised controlled trial. Lancet 388(10042):356-364

2. Watson AJ, Hudson J, Wood J, Kilonzo M, Brown SR, McDonald A, Norrie J, Bruhn H, Cook JA, eTHoS study group (2016) Comparison of stapled haemorrhoidopexy with traditional excisional surgery for haemorrhoidal disease (eTHoS): a pragmatic, multicentre, randomised controlled trial. Lancet 388(10058):2375-2385

3. Giamundo P, Braini A, Calabro G, Crea N, De Nardi P, Fabiano F, Lippa M, Mastromarino A, Tamburini AM (2018) Doppler-guided hemorrhoidal dearterialization with laser (HeLP): a prospective analysis of data from a multicenter trial. Tech Colo. https://doi. org/10.1007/s10151-018-1839-5

4. Schein M (2001) New 'surgical innovation' as a revolution. Dig Surg 18(3):165-166

5. Sandler RS, Peery AF (2018) Rethinking what we know about haemorrhoids. Clin Gastroenterol Hepatol. https://doi. org/10.1016/j.cgh.2018.03.020

6. Pagano $C$, Vergani $C$, Invernizzi $C$, Bussone $M$, Benegiamo $G$, Venturi M (2018) Mucopexy-Recto Anal Lifting (Mu-RAL): a standardized minimally invasive method of managing symptomatic hemorrhoids, with an innovative suturing technique and the HemorPex System ${ }^{\circledR}$. Minerva Chir

7. Grotenhuis BA, Nonner J, de Graaf EJR, Doornebosch PG (2018) Implementation of a new high-volume circular stapler in stapled anopexy for hemorrhoidal disease: is patient's short-term outcome affected by a higher volume of resected tissue? Dig Surg 35(5):406-410

8. Zoulamoglou M, Kaklamanos I, Zarokosta M, Flessas I, Bonatsos V, Piperos T, Theodoropoulos P, Barla G, Stathopoulou I, Mariolis-Sapsakos T (2017) The ligament of Parks as a key anatomical structure for safer hemorrhoidectomy: anatomic study and a simple surgical note. Ann Med Surg (Lond) 16;24:31-33

9. Garg P (2018 Jul) Conservative treatment of hemorrhoids deserves more attention in guidelines and clinical practice. Dis Colon Rectum 61(7):e348

10. van Tol RR, van Zwietering E, Kleijnen J, Melenhorst J, Stassen LPS, Dirksen CD, Breukink SO (2018) Towards a core outcome set for hemorrhoidal disease-a systematic review of outcomes reported in literature. Int J Colorectal Dis 33:849-856

11. van Tol RR, Melenhorst J, Dirksen CD, Stassen LPS, Breukink SO (2017) Protocol for the development of a Core Outcome Set (COS) for hemorrhoidal disease: an international Delphi study. Int J Colorectal Dis 32:1091-1094

12. Shanmugam V, Thaha MA, Rabindranath KS et al (2005) Rubber band ligation versus excisional haemorrhoidectomy for haemorrhoids. Cochrane Database Syst Rev 3:CD005034

13. NHS England (2018) Evidence based interventions. https://www. england.nhs.uk/evidence-based-interventions/. Accessed 15 Aug 2018 